\title{
Interleukin-18 as a Biomarker of Subclinical Lupus Nephritis
}

\author{
Samah Abdel Rahman EL BAKRY, ${ }^{1}$ AbdelAzim Mohamed ALHEFNY, ${ }^{1}$ Dina Shawky AL-ZIFZAF, ${ }^{2}$ \\ Ola Hasan NADA, ${ }^{3}$ Rania Hamdy EL KABARITY, ${ }^{4}$ Khaled OMAR ${ }^{4}$ \\ ${ }^{1}$ Division of Rheumatology, Department of Internal Medicine, Faculty of Medicine, Ain Shams University, Cairo, Egypt \\ ${ }^{2}$ Department of Physical Medicine and Rehabilitation, Faculty of Medicine, Ain Shams University, Cairo, Egypt \\ ${ }^{3}$ Department of Pathology, Faculty of Medicine, Ain Shams University, Cairo, Egypt \\ ${ }^{4}$ Department of Clinical Pathology, Faculty of Medicine, Ain Shams University, Cairo, Egypt
}

\begin{abstract}
Objectives: This study aims to investigate the relationship between serum interleukin-18 (IL-18) and its renal expression with histological classification of lupus nephritis in patients with insignificant proteinuria, and evaluate serum IL-18 as a biomarker of subclinical renal involvement in lupus patients.

Patients and methods: Forty lupus patients ( 5 males, 35 females; mean age $26.3 \pm 7.9$ years; range 16 to 52 years) with proteinuria less than $0.5 \mathrm{~g} / 24$ hours and 20 healthy controls ( 2 males, 18 females, mean age $25.5 \pm 6.3$ years; range 16 to 49 years) were included. Patients underwent full history taking, thorough clinical examination, assessment of disease activity, measurement of serum IL-18, and renal biopsies for histopathological assessment and immunostaining for tissue expression of IL-18.

Results: Lupus patients had significantly higher serum IL-18 levels than controls (612.4 and 209.3 pg/mL, respectively; p<0.01). Serum IL-18 was significantly higher in patients with classes III, IV and V lupus nephritis $(609.1,833.1$ and $790 \mathrm{pg} / \mathrm{mL}$, respectively) than in patients with class I and II ( 370 and $476.8 \mathrm{pg} / \mathrm{mL}$, respectively). Immunostaining of IL-18 showed glomerular expression in classes IV and V, glomerular and tubular infiltration in class III, tubular pattern in class II and no staining in class I. Patients with diffuse glomerular IL-18 staining had higher levels of serum IL-18 than those with no staining $(840.8$ and $522.9 \mathrm{pg} / \mathrm{mL}$, respectively; $\mathrm{p}=0.0)$. Those with diffuse tubular IL- 18 staining had higher levels of serum IL-18 than those with no staining ( 514.2 and $393 \mathrm{pg} / \mathrm{mL}$, respectively; $\mathrm{p}<0.05$ ). Serum IL-18 correlated with serum creatinine and the activity index of renal biopsies.

Conclusion: IL-18 may play a role in the pathogenesis of lupus nephritis. Results of this study showed that serum IL-18 reflects the extent of renal injury in lupus even in absence of significant proteinuria regardless of the level of disease activity; proposing its early role in pathogenesis of lupus nephritis. Thus serum IL-18 can be used as a biomarker that distinguishes the different histological classes of subclinical lupus nephritis.

Keywords: Lupus nephritis; serum IL-18; systemic lupus erythematosus.
\end{abstract}

Systemic lupus erythematosus (SLE) is a chronic autoimmune disease with varied clinical manifestations and severe organ damage. ${ }^{1}$ SLE is a heterogenic disease where $\mathrm{T}$ helper 2 (Th2) is suggested to play an important role in loss of immune tolerance and increased antigenic load with defective B-cell suppression. ${ }^{2}$ Yet, elevation of cytokines related to Th1 and Th2 have been demonstrated in both animal and human models. ${ }^{3}$

Lupus nephritis (LN) is a common, serious clinical feature of SLE that affects about one third of lupus patients. ${ }^{4}$ Patients with LN have increased risk of progressive deterioration of kidney function as well as increased mortality rates. ${ }^{5}$ The pathogenesis of $\mathrm{LN}$ is mostly attributed to glomerular deposition of immune complexes and cytokine imbalance. ${ }^{6}$ Experimental models have shown the important role of Th1 cytokines in the development and progression of LN, where interleukin-12 (IL-12) and IL-18 were found in the glomeruli of human and animal models of LN.7,8

Interleukin-18 is a unique pro-inflammatory cytokine which is a key player in many inflammatory diseases. It strongly induces interferon-gamma 
(IFN- $\gamma$ ) as well as promoting the production of other inflammatory mediators, such as tumor necrosis factor- $\alpha$, and granulocyte macrophage colony stimulating factor (GM-CSF). ${ }^{9}$ Several studies have shown that IL-18 was involved in initiation and progression of many primary and secondary kidney diseases. ${ }^{10-12}$ In lupus patients, IL-18 has been implicated in the pathogenesis, activity and determination of severity of renal involvement. $^{13,14}$ In murine models, overexpression of IL-18 receptor accessory chain on lymphocyte was demonstrated and this led to mounting of IFN- $\gamma$ secretion. Moreover, a relationship between tubular IL-18 expression and renal disease activity has been demonstrated. ${ }^{13,15}$ Tucci et al. ${ }^{16}$ in 2008 demonstrated decrease in peripheral plasmacytoid dendritic cells (DCs), which expressed IL-18 receptor, in patients with LN and they suggested that the high level of expression of IL-18 receptor by peripheral plasmacytoid DCs helps the DCs to relocate within glomeruli under IL-18 stimulation and promote renal damage. Furthermore, Hatef et al. ${ }^{17}$ in 2013 showed a correlation between IL-18 and serum Fas in patients with LN being highly significant in patients with severe SLE than those with milder disease. In addition, the therapy targeted to IL-18 was found to protect mice from LN. ${ }^{18}$

Patients with LN may experience similar clinical manifestations despite having different degrees of renal involvement. Furthermore, patients with moderate levels of proteinurea (>1000 mg/24 h) might have significant renal involvement (Class III, IV or $\mathrm{V}$ LN). That is why renal biopsy in SLE is needed for early and accurate diagnosis of LN and hence early planning of treatment for better patients outcome. ${ }^{19,20}$ Yet, renal biopsy has its limitations and contraindications. That is why new biomarkers are needed to improve the diagnostic accuracy and sensitivity of lupus renal disease, monitoring of treatment response. ${ }^{21}$

In this study, we investigated the relationship between serum IL-18 and its renal expression with histological classification of LN in patients with insignificant proteinuria, and evaluate serum IL-18 as a biomarker of subclinical renal involvement in lupus patients.

\section{PATIENTS AND METHODS}

This cross sectional, case-control, and observational study was conducted on 40 SLE patients ( 5 males, 35 females; mean age $26.3 \pm 7.9$ years; range 16 to 52 years) with proteinuria less than $0.5 \mathrm{~g} / 24$ hours, recruited between October 2011 and December 2012. Patients were diagnosed according to the Updated of the 1982 American College of Rheumatology revised criteria for diagnosis of SLE. ${ }^{22}$ The study also included 20 apparently healthy controls (2 males, 18 females, mean age 25.5 \pm 6.3 years; range 16 to 49 years). Patients with other rheumatic diseases and nephritis due to other causes were excluded as well as patients with proteinuria $>500 \mathrm{mg} / 24 \mathrm{hrs}$ and patients with coexisting causes of renal involvement. All patients were subjected to full history taking. Thorough clinical examination was performed to each patient with special emphasis on symptoms and signs of renal involvement, assessment of the disease activity according to the University of Toronto Systemic Lupus Erythematosus Disease Activity Index (SLEDAI). ${ }^{23}$ All patients were on prednisone (15-25 mg/day), hydroxychloroquine (400 mg/day), and azathioprine (100-150 mg/day). All participants gave written informed consents to participate after receiving full explanation of the study, which was approved by our local ethics committee.

Venous blood $(8 \mathrm{~mL})$ was withdrawn from each patient where, $5 \mathrm{~mL}$ were placed in EDTA tube for performing complete blood count and erythrocyte sedimentation rate (ESR), and $3 \mathrm{~mL}$ of blood were collected in plain vacutainers for analysis of antinuclear antibodies and anti-double stranded DNA (anti-dsDNA). Serum samples were stored at $-20{ }^{\circ} \mathrm{C}$ until time of assay. Complete blood count was done using Coulter counter (T660) (Coulter Electronics Inc., USA), ESR was done by the Westergren method. Antinuclear antibodies and anti-dsDNA antibodies were analyzed using indirect immunofluorescence assay using IMMCO Diagnostics (IMMCO Diagnostics, Inc., NY, USA), (antinuclear antibodies on Hep-2 substrate and anti-dsDNA on crithidialuciliae substrate). Serum complement level assessment (C3 and C4) was performed by Nephelometry (Minineph human Ig kit, Binding site Ltd, Birmingham, UK). Renal function tests including serum creatinine, blood urea, creatinine clearance and routine microscopic 


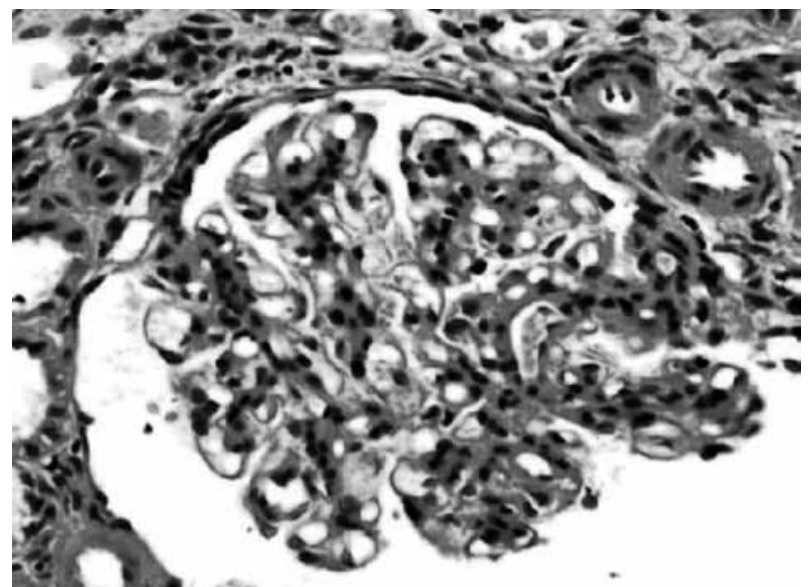

Figure 1. Lupus nephritis class II, mild, global, mesangial hypercellularity with increase in mesangial matrix and thin capillary loops (H-E x 400).

urine analysis for presence of pyuria, hematuria and casts were done. Twenty-four hours urine was collected to assay protein. Liver and kidney function tests were conducted using Synchron CX9 (Beckman instrument Inc., Brea, California, USA).

Quantative analysis of IL-18 was performed using a commercially available enzyme-linked immunosorbent assay kit supplied by Ray Biotech, Inc. (Ray Biotech, Inc., GA, USA), where normal values ranged from $0-732.7 \mathrm{pg} / \mathrm{mL}$.

All patients were performed renal biopsies. The renal tissue obtained was evaluated by light microscopy. The biopsies were graded

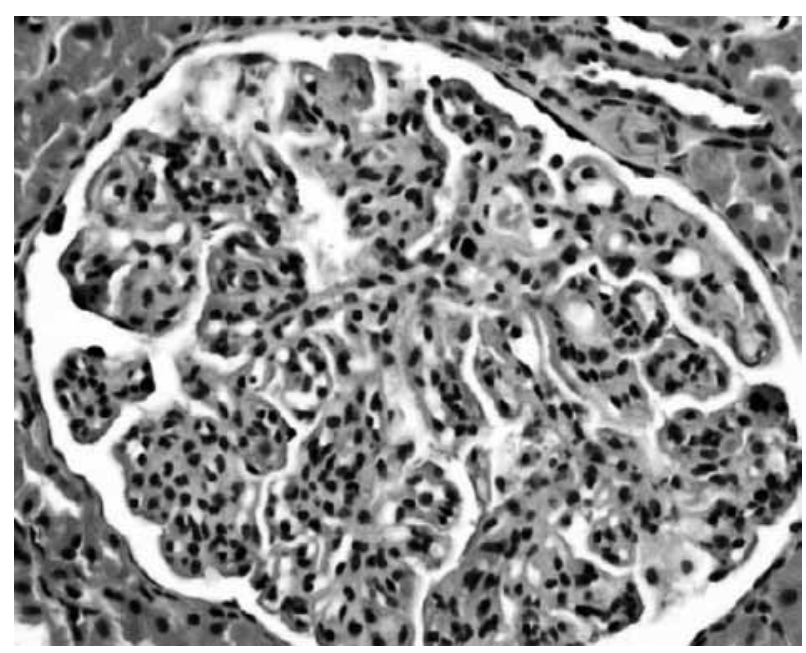

Figure 3. Lupus nephritis class IV, glomerular capillary walls are segmentally thickened by wire-loop deposits and intraluminal deposit form hyaline thrombus (H-E x 400).

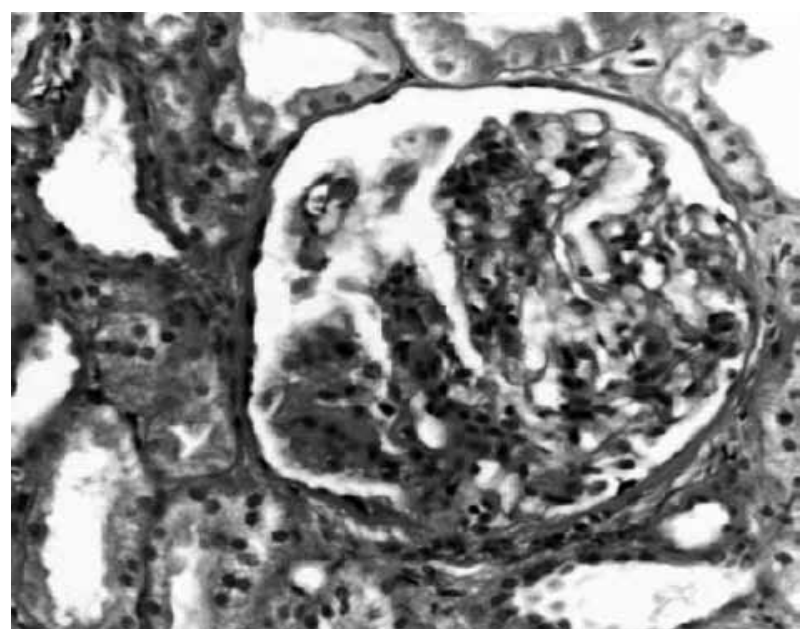

Figure 2. Lupus nephritis class III, prominent endocapillary and mesangial hypercellularity, with "wire loops"; (Periodic-acid Schiff x 400).

according to the classification of $\mathrm{LN}$ by International Society of Nephrology/Renal Pathology Society $2003^{24}$ in which normal glomeruli are designated as class I LN while mesangial hypercellularity represents Class II, and a state of LN showing focal or diffuse segmental or global endo- or extracapillary glomerulonephritis, with or without mesangial alterations is classified as class III (focal LN) and class IV (diffuse LN) respectively. Membranous nephritis is categorized as class $\mathrm{V}$; however, class VI is characterized by advanced sclerosis. Activity and chronicity scores were used according to Austin et al., ${ }^{25} 1983$ (Figures 1-4).

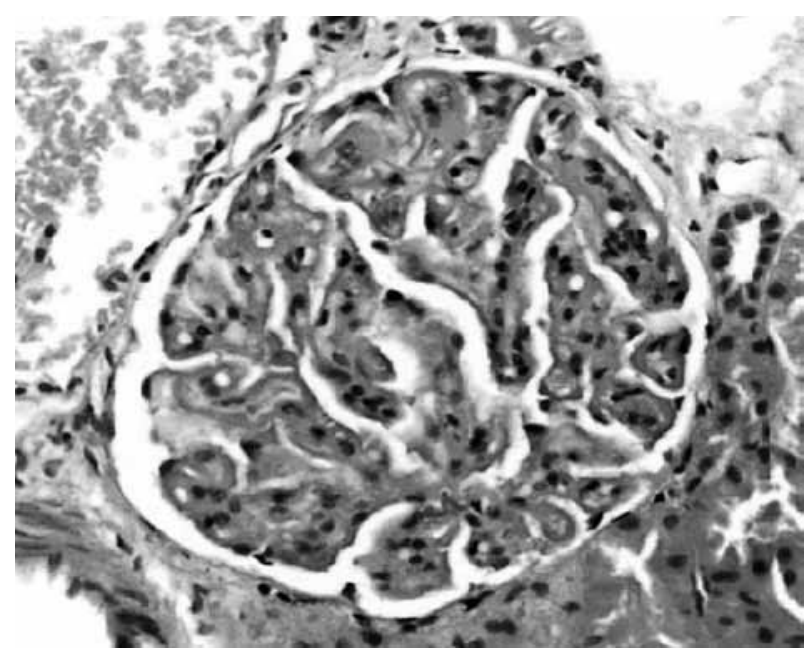

Figure 4. Lupus nephritis class $\mathrm{V}$, regular thickening and rigidity of the glomerular capillary walls accompanied by mesangial hypercellularity (H-E x 400). 
Table 1. The descriptive data of systemic lupus erythematosus patients included in the study

\begin{tabular}{lcc}
\hline & Mean \pm SD & Min.-Max. \\
\hline Age (years) & $26.3 \pm 8.0$ & $16-52$ \\
Disease duration (months) & $9.6 \pm 1.6$ & $7.0-12.0$ \\
White blood cells $\left(10^{3} / \mathrm{mm}^{3}\right)$ & $6.4 \pm 3.4$ & $1.5-14.8$ \\
Neutrophils & $45.8 \pm 10.1$ & $23-58$ \\
Total lymphocyte count $\left(\mathrm{mm}^{3}\right)$ & $19.1 \pm 4.7$ & $11-27$ \\
Hemoglobin (gm/dL) & $9.6 \pm 1.5$ & $5.9-12.4$ \\
Mean corpuscular volume & $81.6 \pm 10.2$ & $67-125$ \\
Mean corpuscular hemoglobin & $27.0 \pm 5.3$ & $20-55$ \\
Platelets (103/mm ${ }^{3}$ ) & $198.3 \pm 108.4$ & $43-484$ \\
Erythrocyte sedimentation rate (mm first hour) & $90.9 \pm 36.6$ & $20-140$ \\
Alanine transaminase & $29.9 \pm 36.3$ & $6-157$ \\
Aspartate aminotransferase & $30.6 \pm 35.1$ & $10-189$ \\
Total protein & $6.9 \pm 0.8$ & $4.7-8.9$ \\
Albumin & $3.3 \pm 0.4$ & $2-4$ \\
Total billirubin & $0.7 \pm 0.3$ & $0.2-1.2$ \\
Direct billirubin & $0.1 \pm 0.1$ & $0-0.4$ \\
Sodium & $137.4 \pm 4.1$ & $128-150$ \\
Potasium & $3.9 \pm 0.4$ & $3.4-5$ \\
Serum creatinine (mg/dL) & $0.6 \pm 0.2$ & $0.3-1.1$ \\
Blood urea nitrogen & $13.5 \pm 5.6$ & $4-30$ \\
Complement 3 (mg/dL) & $39.8 \pm 35.2$ & $7-140$ \\
Complement 4 (mg/dL) & $15.6 \pm 8.9$ & $5-40$ \\
Protein creatinine ratio in urine & $0.2 \pm 0.1$ & $0-0.4$ \\
Creatinine clearance (mL/min) & $96.1 \pm 15.9$ & $45-121$ \\
Prothombin time & $11.9 \pm 1.1$ & $11-14$ \\
Partial thromboblastin time & $34.1 \pm 3.1$ & $23-43$ \\
International normalized ratio & $1.0 \pm 0.1$ & $0.8-1.1$ \\
Systemic lupus erythematosus disease activity index score & $13.7 \pm 4.3$ & $7-23$ \\
\hline SD: Standard deviation; Min.: Minimum; Max.: Maximum. & & \\
& &
\end{tabular}

Immunohistochemical staining was performed at room temperature using immunostainer (Shandon Sequenza Immunostainer). Formaldehyde fixed paraffinembedded serial sections of renal biopsies were cut at 4- $\mu \mathrm{m}$ thickness and mounted onto Superfrost plus slides. Sections were dewaxed and hydrated in graded ethanol prior to antigen retrieval by microwaving in $0.01 \mathrm{M}$ citrate buffer ( $\mathrm{pH}$ 6.0) for 6 minutes. Endogenous peroxidase activity was inactivated with $3 \% \mathrm{H}_{2} \mathrm{O}_{2}$ in methanol for 20 minutes. Sections were treated with $5 \%$ goat serum in Tris-buffered saline to block nonspecific binding. Goat anti-human IL-18 (R\&D Systems, Abingdon, United Kingdom) was added at a dilution of 1:200 (final concentration of $0.5 \mathrm{mug} / \mathrm{mL}$ ) and sections were incubated overnight at $4{ }^{\circ} \mathrm{C}$. The biotinylated conjugate and streptavidin peroxidase were applied for 15 minutes, and diaminobenzidine chromogen was used as a peroxidase substrate complex (all from DAKO labelled streptavidin biotin + kit peroxidase, DAKO Copenhagen, Denmark). Tissue sections were then counterstained with hematoxylin for 10 seconds, dried and mounted with aqueous mounting medium (DAKO Copenhagen, Denmark). Intrinsic positive controls for immunoreactivity in each section were IL-18 stained cells in lymphoid follicles. Each series of sections contained negative controls without primary antibodies. In addition, a control using immunoglobulin $G$ from non-immunized goats ( $R \& D$ systems) at the same concentration as that of anti-IL-18 immunoglobulin $G$ was carried out in a number of tissue sections that excluded nonspecific binding. The renal tissues

Table 2. The frequency of various clinical presentations among systemic lupus erythematosus patients

\begin{tabular}{lcc}
\hline Clinical variable & $\mathrm{n}$ & Frequency \% \\
\hline Fever & 8 & 20 \\
Photosensitivity & 27 & 67.5 \\
Malar rash & 35 & 87.5 \\
Oral ulcer & 26 & 65 \\
Alopecia & 7 & 17.5 \\
Arthralgia & 38 & 95 \\
Recurrent thrombosis & 1 & 2.5 \\
Serositis & 2 & 5 \\
Central nervous system disorders & 1 & 2.5 \\
Anti-dsDNA positivity & 36 & 90 \\
dsDNA: Double stranded deoxyribonucleic acid. & \\
\hline
\end{tabular}




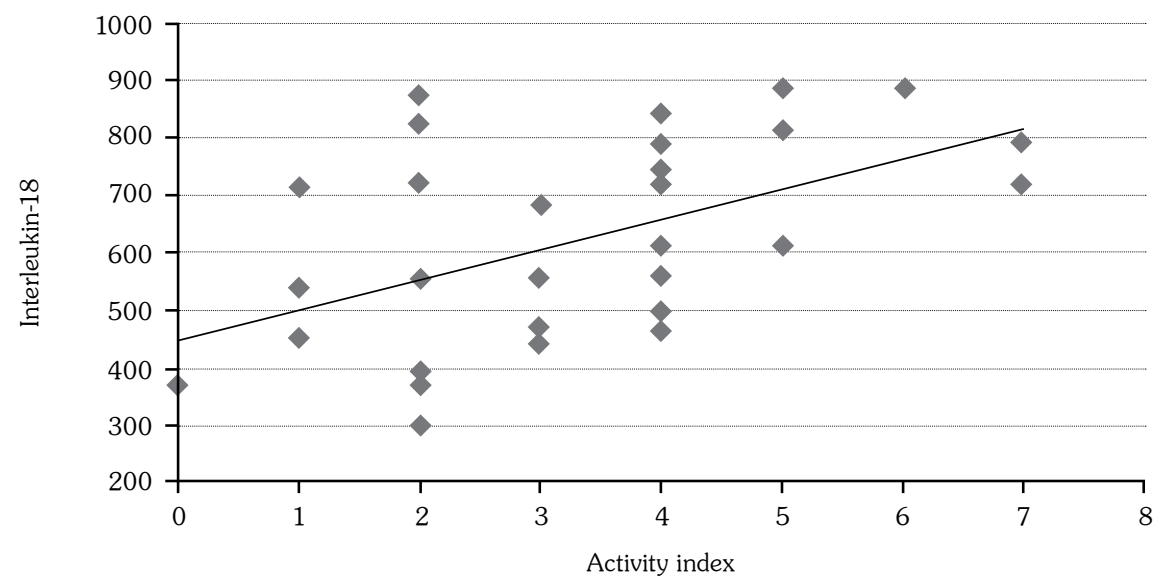

Figure 5. Correlation between serum interleukin-18 and activity index of renal biopsy.

immunostained for IL-18 were assessed without knowledge of the clinicopathologic features. Samples were considered positive when the unequivocal strong brown colored immunostaining of the cytoplasm was seen in more than $10 \%$ of the glomerular or tubular cells whether the staining was focal or diffuse. However, a faint staining was designed as negative. ${ }^{26,27}$

\section{Statistical analysis}

Statistical analysis was performed using SPSS for Windows version 10.0 software program (SPSS Inc., Chicago, IL, USA). Descriptive statistics were expressed in the form of mean, standard deviation, minimum, maximum, and range of numerical data as well as frequency and percentage of non-numerical data. Student's t test was used to test the difference in mean values of continuous variables, and Chi-square test to compare two groups regarding non-numerical variables. Correlation (Pearson correlation coefficient $r$ ) assessing strength and direction of the linear relationship between two variables was shown, and one-way ANOVA test $(\mathrm{F})$ was used to test the difference between more than two means. Diagnostic validity tests were calculated.

\section{RESULTS}

Descriptive laboratory and clinical data of SLE patients are displayed in Tables 1 and 2 . SLE patients had a significantly higher serum IL-18 level $(612.4 \pm 169.6 \mathrm{pg} / \mathrm{mL})$ than controls $(209.3 \pm 92.3 \mathrm{pg} / \mathrm{mL})(z=-6.234, p<0.01)$. Serum
IL-18 showed significant positive correlation with serum creatinine and blood urea nitrogen (BUN) of our patients $(r=0.462, p=0.003$ and $r=0.428$, $p=0.006$, respectively), while it did not correlate significantly with the disease activity assessed by the SLEDAI ( $r=0.004, p>0.01)$ nor with any of the other laboratory data of the patients nor with the dose of prednisone or azathioprine.

Histopathological examination of the 40 renal tissue sections included in our study showed that two cases (5\%) were class I, 12 cases (30\%) were class II, 16 cases (40\%) were class III, nine cases (22.5\%) were class IV, while class $\mathrm{V}$ was only represented by one case $(2.5 \%)$. The mean values for the activity and chronicity indices were as follows; $2.42,3.19$ and 4.44 mean activity score for classes II, III, IV, respectively and 2.92, 3.11 and 2.5 mean chronicity score for classes II, III, IV, respectively. Serum IL-18 showed significant positive correlation with the activity index of the renal biopsies $(r=0.534, p<0.001)$ (Figure 5). It also demonstrated significant increase among the different classes of glomerulonephritis (Table 3 and Figure 6).

Interleukin-18 immunoexpression is designated as brown granular cytoplasmic immunostaining. IL-18 immunoreactivity was observed in the epithelium of renal tubules in $75 \%$ of class II cases and $100 \%$ of classes III, IV and V. IL-18 immunostaining also showed glomerular expression in $50 \%$ of class II cases, $62.5 \%$ of class III cases and $100 \%$ of both classes IV and V (Figures 7-9). Meanwhile, immunostaining was neither glomerular nor tubular in class I. A highly 


\begin{tabular}{|c|c|c|}
\hline & & Serum IL-18 $(\mathrm{pg} / \mathrm{mL})$ \\
\hline & Number of patients & Mean \pm SD \\
\hline \multicolumn{3}{|l|}{ Class } \\
\hline I & 2 & $370 \pm 0$ \\
\hline II & 12 & $476.8 \pm 95.8$ \\
\hline III & 16 & $609.2 \pm 112.9$ \\
\hline IV & 9 & $833.1 \pm 51.8$ \\
\hline $\mathrm{V}$ & 1 & 790 \\
\hline
\end{tabular}

statistically significant association was established between different types of tubular and glomerular expression of IL-18 among different LN classes and the corresponding serum IL-18. Patients with focal tubular distribution had less serum IL-18 than those with diffuse focal distribution, and patients with focal glomerular distribution had significantly lower levels of serum IL-18 than those with diffuse glomerular staining $(p=0.013$ and $\mathrm{p}=0.00$, respectively) (Tables 4 and 5).

To confirm the role of IL-18 in detecting the severity of renal involvement as determined by renal biopsy, patients were divided into two groups according to the 2003 classification of LN. Group 1 with class I and II renal glomerulonephritis showed significantly lower serum IL-18 than group 2 of SLE patients with class III, IV and V (461.6 \pm 96.3 $\mathrm{pg} / \mathrm{m}$; and $693.7 \pm 142.6 \mathrm{pg} / \mathrm{mL}$, respectively; $p=0$ ) (Figure 10). None of the renal function tests measured in this study (serum creatinine, BUN,

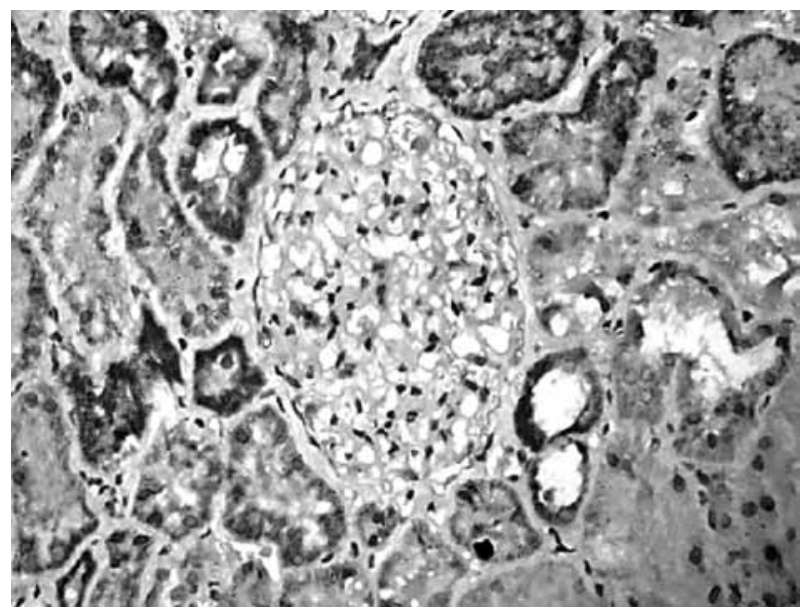

Figure 7. Renal tissue of a patient with class II lupus nephritis showing strong tubular and no glomerular staining of interleukin-18 (I.P. x 400).

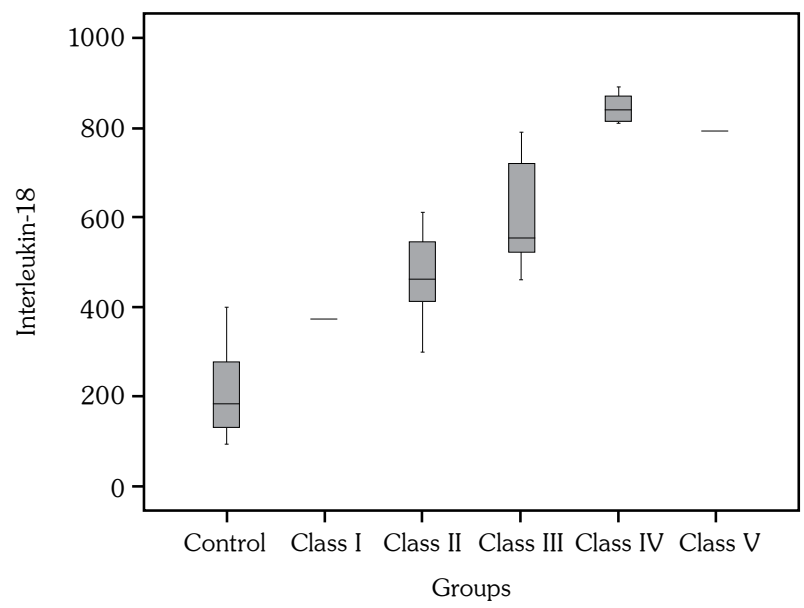

Figure 6. Plot chart of serum interleukin- 18 among the different nephritis classes and controls.

creatinine clearance and protein-creatinine ratio) showed significant difference among these two groups of SLE patients.

\section{DISCUSSION}

Systemic lupus erythematosis is a systemic autoimmune disease with multi-organ affection and diversity of clinical presentations as well as unpredictable course. ${ }^{28}$ Kidney disease is common in course of SLE and may occur at any time, presenting a central and serious complication with glomerulonephritis as a

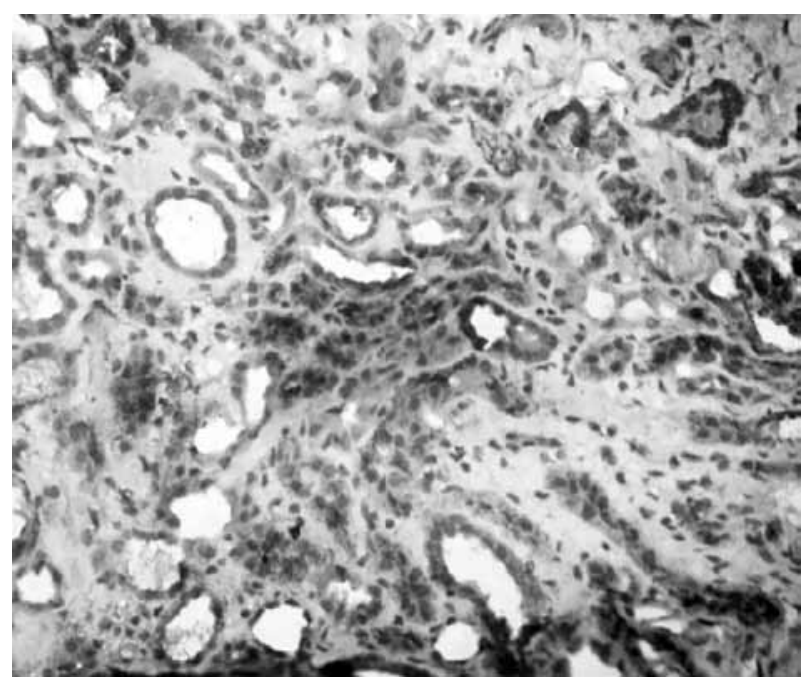

Figure 8. Renal tissue of a patient with class III lupus nephritis showing diffuse tubular and faint focal glomerular staining of interleukin-18 (I.P. x 400). 


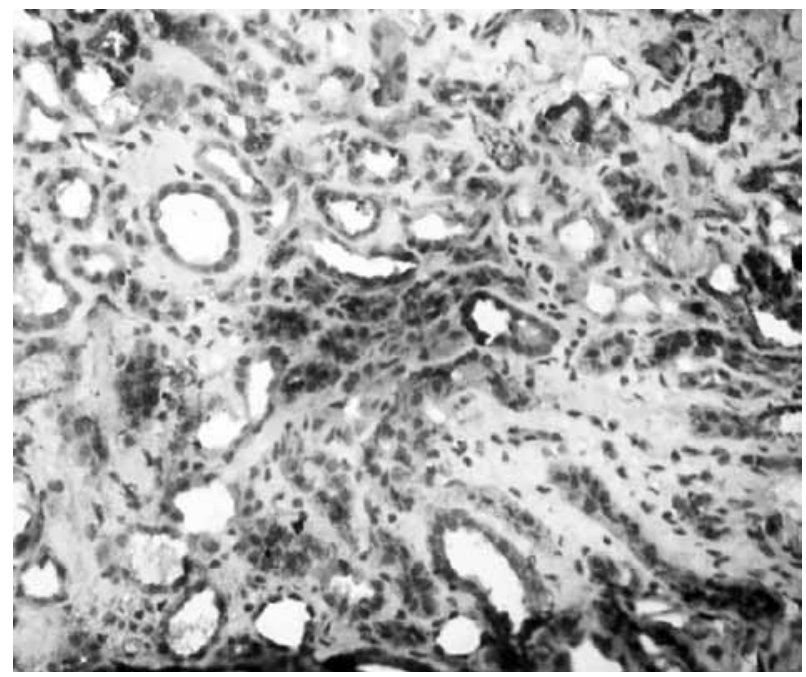

Figure 9. Renal tissue of a patient with class IV lupus nephritis showing strong focal tubular staining of interleukin-18 (I.P. x 400).

major cause of morbidity and mortality in patients with SLE. ${ }^{29,30}$ Pathogenesis of LN involves many players. One of these players is overproduction of Th1 cytokines, among which is IL-18.

In the current work, we studied the serum level and renal expression of IL-18 in lupus patients with insignificant proteinuria. Serum IL-18 was significantly higher in SLE patients than controls. This finding agrees with the results reported by other research groups., ${ }^{1,14,31-33}$ It showed significant positive correlation with serum creatinine and BUN. IL-18 being an important regulator of Th1 responses has a role in the pathogenesis of LN as it stimulates production of pro-inflammatory cytokines such as TNF- $\alpha$ and IL-1 $\beta$ in Th1 cells, and macrophages, upregulates chemokine production and expression of adhesion molecules, enhances perforin- and FasL-mediated cytotoxicity by NK and T cells, and induces the release of matrix metalloproteinases, which contributes to the inflammatory reaction and subsequent tissue damage. ${ }^{14}$

In our study, IL-18 did not correlate significantly with the disease activity assessed by the SLEDAI nor with any of the other laboratory data of the patients. This was not in concordance with Migliorini et al. ${ }^{14}$ who found that total and free serum IL-18 levels were higher in patients with active disease (with nephritis or active non-renal disease), while urinary levels of total and free IL-18 did not correlate with disease activity, nor with other reports. ${ }^{31,34,35}$ This difference in reports could be due to the fact that non of our lupus patients had inactive disease and they all ranged from mild to severe activity with a mean SLEDAI of $13.7 \pm 4.3$ which made it difficult to correlate the IL-18 level with the whole spectrum

Table 4. Comparison between serum interleukin-18 levels among the different systemic lupus erythematosus patients divided according to degree of interleukin-18 tubular staining after renal biopsy

\begin{tabular}{lccccr}
\hline & & \multicolumn{2}{c}{ Serum IL-18 } & & \\
\cline { 3 - 5 } Degree of IL-18 tubular staining & Number of patients & Mean \pm SD & H & $p$ & Sig \\
\hline Negative & 5 & $393 \pm 69.6$ & 22.13 & 0.0 & HS \\
Faint focal & 16 & $599.3 \pm 152.0$ & & & \\
Focal & 13 & $760.2 \pm 121.9$ & & & \\
Diffuse & 6 & $514.2 \pm 69.7$ & & & \\
\hline IL-18: Interleukin-18; SD: Standard deviation; Sig: Significance; HS: Highly significant. & & & \\
\hline
\end{tabular}

Table 5. Comparison between serum interleukin-18 levels among the different systemic lupus erythematosus patients divided according to degree of interleukin- 18 glomerular staining after renal biopsy

\begin{tabular}{|c|c|c|c|c|c|}
\hline \multirow[b]{2}{*}{ Degree of IL-18 glomerular staining } & \multirow[b]{2}{*}{ Number of patients } & \multirow{2}{*}{$\frac{\text { Serum IL-18 }}{\text { Mean } \pm \text { SD }}$} & \multirow[b]{2}{*}{$\mathrm{H}$} & \multirow[b]{2}{*}{$p$} & \multirow[b]{2}{*}{ Sig } \\
\hline & & & & & \\
\hline Negative & 12 & $522.9 \pm 115.6$ & 17.03 & 0.001 & HS \\
\hline Faint focal & 11 & $486.5 \pm 112.3$ & & & \\
\hline Focal & 7 & $676.8 \pm 136.1$ & & & \\
\hline Diffuse & 10 & $840.9 \pm 35.1$ & & & \\
\hline
\end{tabular}




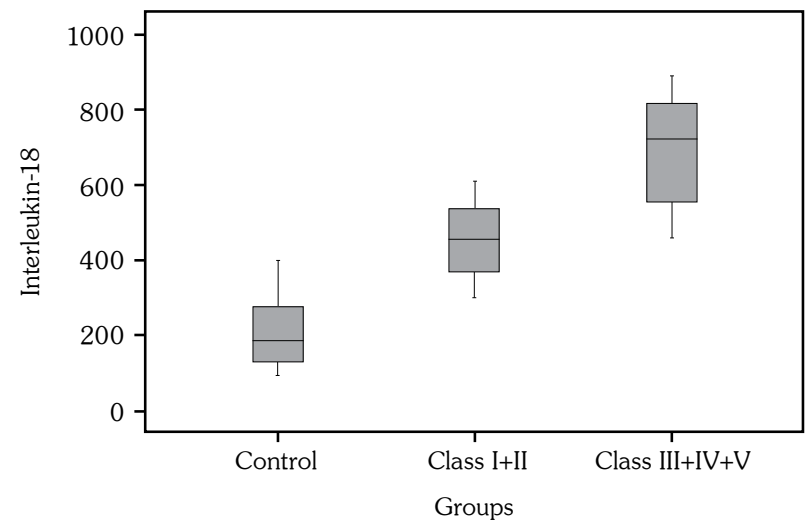

Figure 10. Plot chart of serum interleukin-18 among two patient groups and controls.

of activity.

Unfortunately, similar clinical manifestations are observed among SLE patients in spite of different classes of $\mathrm{LN}$, and considerable renal involvement (class III, IV, or V LN) can occur in SLE patients with insignificant proteinuria $\left(<0.5 \mathrm{gm} / 24\right.$ hours). ${ }^{36,37}$ Histopathological examination of the 40 renal biopsies of patients included in our study showed that two patients (5\%) had class I, 12 (30\%) had class II, 16 (40\%) had class III, nine (22.5\%) had class IV and only one patient (2.5\%) had class $\mathrm{V}$ LN.

Local production of IL-18 has been reported to play an important role in driving the migration of dendritic cells to the kidney with attraction of more inflammatory cells and maintenance of kidney inflammation. ${ }^{6,38}$ Data from several studies suggested that up-regulation of IL-18 in animal models and humans, is associated with nephritic kidneys and related to the degree of renal inflammation. ${ }^{33,39}$ In our study, we found that serum levels of IL-18 showed significant increase among the different classes of glomerulonephritis, being higher in class IV and V LN than in class III and II LN. We have also demonstrated that serum IL-18 correlated significantly with the activity index of the renal biopsies.

Testing for serum IL-18 helped us to differentiate between milder (class I and II) severe (class III, IV and $\mathrm{V}$ ) forms of LN where all renal functions tested showed no significant differences between all forms of LN.

As well, renal expression of IL-18 was valuable. IL-18 was present in the epithelium of renal tubules in $75 \%$ of class II cases and $100 \%$ of classes III, IV and V where it showed glomerular expression in $50 \%$ of class II cases, $62.5 \%$ of class III cases and 100\% of both classes IV and $\mathrm{V}$ (Figures 7-9). These findings corroborate the findings of Faust et al., ${ }^{13}$ who described marked expression of IL-18 in nephritic kidneys from Fas mice lpr that was not confined to interstitial or glomerular infiltrating mononuclear cells, but was also detected in tubular epithelial cells. They attributed elevated circulating levels of IL-18 in part to the local IL-18 production within the kidneys. Their further in vitro studies suggested that up regulation of renal IL-18 is a prerequisite for the local inflammatory cytokine cascade especially that the interaction between TNF and IL-18 might provide a positive-amplification loop that accelerates the auto-immune kidney disease. In addition to a possible role of IL-18 in the induction of apoptosis in tubular epithelial cells, ${ }^{13}$ Shui et al. ${ }^{40}$ also demonstrated IL-18 overexpression, associated with hyperplasia and apoptosis of mesangial cells in the glomeruli of lipopolysaccharid-accelerated LN mice. They suggested that local IL-18 in the nephritic kidneys could act like a paracrine cytokine to exacerbate glomerular inflammation and is probably involved in the apoptosis of the downstream tubular cells. The increased glomerular expression of IL-18 in established human LN was also reported by different groups.,16,33,41 To our knowledge, we are the first group to report expression of IL-18 in renal tissue of subclinical LN.

The increased expression in renal tissue was accompanied by an increase in serum IL-18 that showed statistically significant difference among the different types of both glomerular and tubular expression (whether faint focal, focal or diffuse). These findings verify that serum IL-18 can successfully mirror the tissue affection in the kidneys rendering it a probable biomarker of early renal involvement in LN even in the absence of significant proteinuria.

In conclusion, IL-18 may play an important role in the pathogenesis of LN. Data present in this work showed that serum IL-18 reflects the extent of renal injury in lupus even in absence of significant proteinuria regardless of the level of disease activity; proposing its early role in pathogenesis of LN via both local and systemic sources. Thus, IL-18 can be used as a biomarker 
that distinguishes the different histological classes of subclinical LN. One limitation of our study is the relatively small sample size and the lack of follow-up of the change of serum IL-18 levels in response to treatment. Therefore, further studies on larger cohorts are needed to confirm our findings.

\section{Declaration of conflicting interests}

The authors declared no conflicts of interest with respect to the authorship and/or publication of this article.

\section{Funding}

The authors received no financial support for the research and/or authorship of this article.

\section{REFERENCES}

1. Kahlenberg JM, Thacker SG, Berthier CC, Cohen CD, Kretzler M, Kaplan MJ. Inflammasome activation of IL-18 results in endothelial progenitor cell dysfunction in systemic lupus erythematosus. J Immunol 2011;187:6143-56.

2. Akahoshi M, Nakashima $\mathrm{H}$, Tanaka $\mathrm{Y}$, Kohsaka T, Nagano S, Ohgami E, et al. Th1/Th2 balance of peripheral $\mathrm{T}$ helper cells in systemic lupus erythematosus. Arthritis Rheum 1999;42:1644-48.

3. Foster MH, Kelley VR. Lupus nephritis: update on pathogenesis and disease mechanisms. Semin Nephrol 1999;19:173-81.

4. Mok CC. Prognostic factors in lupus nephritis. Lupus 2005;14:39-44.

5. van der Vlag J, Berden JH. Lupus nephritis: role of antinucleosome autoantibodies. Semin Nephrol 2011;31:376-89.

6. Tucci M, CiavarellaS, Strippoli S, DammaccoF, Silvestris F. Oversecretion of cytokines and chemokines in lupus nephritis is regulated by intraparenchymal dendritic cells: a review. Ann N Y Acad Sci 2009;1173:449-57.

7. Calvani N, Richards HB, Tucci M, Pannarale G, Silvestris F. Up-regulation of IL-18 and predominance of a Th1 immune response is a hallmark of lupus nephritis. Clin Exp Immunol 2004;138:171-8.

8. Tucci M, Lombardi L, Richards HB, Dammacco F, Silvestris F. Overexpression of interleukin-12 and $T$ helper 1 predominance in lupus nephritis. Clin Exp Immunol 2008;54:247-54.

9. Dinarello CA. Interleukin-18 and the pathogenesis of inflammatory diseases. Semin Nephrol 2007;27:98114.

10. Araki S, Haneda M, Koya D, Sugimoto T, Isshiki K, Chin-Kanasaki M, et al. Predictive impact of elevated serum level of IL-18 for early renal dysfunction in type 2 diabetes: an observational follow-up study.
Diabetologia 2007;50:867-73.

11. Kitching AR, Turner AL, Wilson GR, Semple T, Odobasic D, Timoshanko JR, et al. IL-12p40 and IL-18 in crescentic glomerulonephritis: IL-12p40 is the key Th1-defining cytokine chain, whereas IL-18 promotes local inflammation and leukocyte recruitment. J Am Soc Nephrol 2005;16:2023-33.

12. Menke J, Bork T, Kutska B, Byrne KT, Blanfeld M, Relle $\mathrm{M}$, et al. Targeting transcription factor Stat 4 uncovers a role for interleukin-18 in the pathogenesis of severe lupus nephritis in mice. Kidney Int 2011;79:452-63.

13. Faust J, Menke J, Kriegsmann J, Kelley VR, Mayet WJ, Galle PR, et al. Correlation of renal tubular epithelial cell-derived interleukin-18 up-regulation with disease activity in MRL-Faslpr mice with autoimmune lupus nephritis.Arthritis Rheum 2002;46:3083-95.

14. Migliorini P, Anzilotti C, Pratesi F, Quattroni P, Bargagna M, Dinarello CA, et al. Serum and urinary levels of IL-18 and its inhibitor IL-18BP in systemic lupus erythematosus. Eur Cytokine Netw 2010;21:264-71.

15. Neumann D, Del Giudice E, Ciaramella A, Boraschi $\mathrm{D}$, Bossù $\mathrm{P}$. Lymphocytes from autoimmune MRL lpr/ lpr mice are hyperresponsive to IL-18 and overexpress the IL-18 receptor accessory chain. J Immunol 2001;166:3757-62.

16. Tucci M, Quatraro C, Lombardi L, Pellegrino C, Dammacco F, Silvestris F. Glomerular accumulation of plasmacytoid dendritic cells in active lupus nephritis: role of interleukin-18. Arthritis Rheum 2008;58:251-62.

17. Hatef MR, Sahebari M, Rezaieyazdi Z, Nakhjavani MR, Mahmoudi M. Stronger Correlation between Interleukin 18 and Soluble Fas in Lupus Nephritis Compared with Mild Lupus. ISRN Rheumatol 2013;2013:850851.

18. Bossù $P$, Neumann D, Del Giudice E, Ciaramella A, Gloaguen I, Fantuzzi G, et al. IL-18 cDNA vaccination protects mice from spontaneous lupus-like autoimmune disease. Proc Natl Acad Sci U S A 2003;100:14181-6.

19. Hsieh YP, Wen YK, Chen ML. The value of early renal biopsy in systemic lupus erythematosus patients presenting with renal affection. Clin Nephrol 2012;77:18-24.

20. Christopher-Stine L, Siedner M, Lin J, Haas M, Parekh H, Petri $M$, et al. Renal biopsy in lupus patients with low levels of proteinuria. J Rheumatol 2007;34:332-5.

21. Mok CC. Biomarkers for lupus nephritis: a critical appraisal. J Biomed Biotechnol 2010;2010:638413.

22. Hochberg MC. Updating the American College of Rheumatology revised criteria for the classification of systemic lupus erythematosus. Arthritis Rheum 1997;40:1725.

23. Bombardier C, Gladman DD, Urowitz MB, Caron $\mathrm{D}$, Chang $\mathrm{CH}$. Derivation of the SLEDAI. A disease activity index for lupus patients. The Committee on Prognosis Studies in SLE. Arthritis Rheum 1992;35:630-40.

24. Weening JJ, D'Agati VD, Schwartz MM, Seshan SV, 
Alpers CE, Appel GB, et al. The classification of glomerulonephritis in systemic lupus erythematosus revisited. J Am Soc Nephrol 2004;15:241-50.

25. Austin HA, Muenz LR, Joyce KM, Antonovych TA, Kullick ME, Klippel $\mathrm{JH}$, et al. Prognostic factors in lupus nephritis. Contribution of renal histologic data. Am J Med 1983;75:382-91.

26. Okamoto M, Azuma K, Hoshino T, Imaoka $\mathrm{H}$, Ikeda J, Kinoshita T, et al. Correlation of decreased survival and IL-18 in bone metastasis. Intern Med 2009;48:763-73.

27. Ye ZB, Ma T, Li H, Jin XL, Xu HM. Expression and significance of intratumoral interleukin-12 and interleukin-18 in human gastric carcinoma. World $\mathrm{J}$ Gastroenterol 2007;13:1747-51.

28. Tarr T, Lakos G, Bhattoa HP, Szegedi G, Shoenfeld Y, Kiss E. Primary anti-phospholipid syndrome as the forerunner of systemic lupus erythematosus. Lupus 2007;16:324-8.

29. Gigante A, Gasperini ML, Afeltra A, Barbano B, Margiotta D, Cianci R, et al. Cytokines expression in SLE nephritis. Eur Rev Med Pharmacol Sci 2011;15:15-24.

30. Manson JJ, Ma A, Rogers P, Mason LJ, Berden $\mathrm{JH}$, van der Vlag $\mathrm{J}$, et al. Relationship between anti-dsDNA, anti-nucleosome and anti-alpha-actinin antibodies and markers of renal disease in patients with lupus nephritis: a prospective longitudinal study. Arthritis Res Ther 2009;14:11:R154.

31. Koenig KF, Groeschl I, Pesickova SS, Tesar V, Eisenberger U, Trendelenburg M. Serum cytokine profile in patients with active lupus nephritis. Cytokine 2012;60:410-6.

32. Wong CK, Ho CY, Li EK, Tam LS, Lam CW. Elevated production of interleukin-18 is associated with renal disease in patients with systemic lupus erythematosus.
Clin Exp Immunol 2002;130:345.

33. Hu D, Liu X, Chen S, Bao C. Expressions of IL-18 and its binding protein in peripheral blood leukocytes and kidney tissues of lupus nephritis patients. Clin Rheumatol 2010;29:717.

34. Park MC, Park YB, Lee SK. Elevated interleukin-18 levels correlated with disease activity in systemic lupus erythematosus. Clin Rheumatol 2004;23:225.

35. Liu X, Bao C, Hu D. Elevated interleukin-18 and skewed Th1:Th2 immune response in lupus nephritis. Rheumatol Int 2012;32:223-9.

36. Al-Hefny AM, El-Bakry SA, Mobasher SA, Abaza N, Nada OH. Renal Biopsy Findings In Lupus Patient With Insignificant Proteinuria: Relation To Disease Activity And Clinical Manifestations. Life Sci J 2013;10:1872-9.

37. Wen YK. Renal biopsy findings in new-onset systemic lupus erythematosus with clinical renal disease. Int Urol Nephrol 2011;43:801-6.

38. Tucci M, Stucci S, Strippoli S, Silvestris F. Cytokine overproduction, T-cell activation, and defective T-regulatory functions promote nephritis in systemic lupus erythematosus. J Biomed Biotechnol 2010;2010:457146.

39. Shimizu C, Fujita T, Fuke Y, Ito K, Satomura A, Matsumoto K, et al. High circulating levels of interleukin-18 binding protein indicate the severity of glomerular involvement in systemic lupus erythematosus. Mod Rheumatol 2012;22:73-9.

40. Shui HA, Ka SM, Wu WM, Lin YF, Hou YC, Su LC, et al. LPS-evoked IL-18 expression in mesangial cells plays a role in accelerating lupus nephritis. Rheumatology 2007;46:1277-84.

41. Chan RW, Lai FM, Li EK, Tam LS, Chow KM, Lai $\mathrm{KB}$, et al. Intrarenal cytokine gene expression in lupus nephritis. Ann Rheum Dis 2007;66:886-92. 